\title{
Correlative Imaging of Tissues: The Potential of Large Volume Array Tomography
}

Irene U. Wacker ${ }^{1,2}$

${ }^{1}$ Institute for Biological Interfaces I, Karlsruhe Institute of Technology, Karlsruhe, Germany

${ }^{2}$ HEiKA, Heidelberg Karlsruhe Research Partnership, Correlative Imaging Platform, Germany

This tutorial will compare different techniques for high resolution imaging of tissue such as array tomography (AT) [1], serial blockface scanning electron microscopy (SBFSEM) [2], and focussed ion beam scanning electron microscopy (FIBSEM) [3]. For AT a biological sample is fixed, either by conventional chemical fixation or by high pressure freezing and freeze substitution, and embedded in resin. Ultrathin serial sections are cut from the block and placed on solid substrates which can be e.g. silicon wafers or glass slides. This array can then be imaged in a SEM or - depending on the choice of resin for embedding - in a light microscope (LM) either directly or after various rounds of antibody labelling. Images have to be aligned and a volume reconstructed before segmentation and volumetric analyses are performed. Since the same array can be imaged in both, LM and SEM, correlation of both modalities is relatively easy. Such a general workflow for AT is shown in Fig. 1 (center and right side).

SBFSEM works directly on a resin block by repeated cycles of imaging its blockface and shaving off thin slivers of material with an ultramicrotome inside the chamber of the SEM. In a FIBSEM an ion beam is used instead of a knife to sequentially remove a few nanometer thin layer of material from the blockface, which is imaged between two milling/shaving actions. Because a stationary block face is imaged both methods create image stacks, which are already aligned. Due to the destructive nature of those two methods - the material removed from the blockface is lost - a correlative workflow is currently only possible by LM imaging of the tissue before working on it in the SEM (as e.g. described in [4]). This requires either genetically encoded markers to be present in the sample and to be retained through the whole of the preparation protocol. Alternatively some other kind of pre-embedding labelling needs to be applied, such as antibody labelling, introduction of fluorescent dyes during freeze substitution [4], or e.g. introduction of neuronal tracers by microinjection.

Another point in the context of "destructive" serial imaging (i.e. SBFSEM and FIBSEM) versus AT is the fact that an array can be regarded as "hardware storage" of the biological sample. It lends itself to hierarchical data recording: Large areas can be imaged at low resolution to create a coarse map of the sample from which the interesting regions can be identified and re-imaged at the desired resolution in a second step. The first round can either be LM (wide field fluorescence, STORM [5], reflection LSM) or low magnification SEM, depending on preparation and labelling.

A number of examples will be discussed to illustrate how different biological questions ask for variations on the general theme of AT. There are several options for many parameters during a complete workflow from the initial preparation of the tissue through staining/labelling and choice of embedding resin to finally the choice of substrate the sections are deposited on. 
References:

[1] KD Micheva, DJ Smith, Neuron 55 (2007), p25-36. Erratum in: Neuron 55 (2007), p. 824.

[2] W Denk, H Horstmann, PLoS Biol. 2 (2004) e329.

[3] JAW Heyman et al., J. Struct. Biol. 155 (2006), p. 63.

[4] MS Lucas et al., in "Methods in Cell Biology" Vol. 111 (Elsevier Inc., Amsterdam) (2012), p.325.

[5] S Nanguneri et al., PLoS ONE. 7 (2012), e38098.

[6] see also Wacker et al., this conference, Symposium A04, for a further case study.

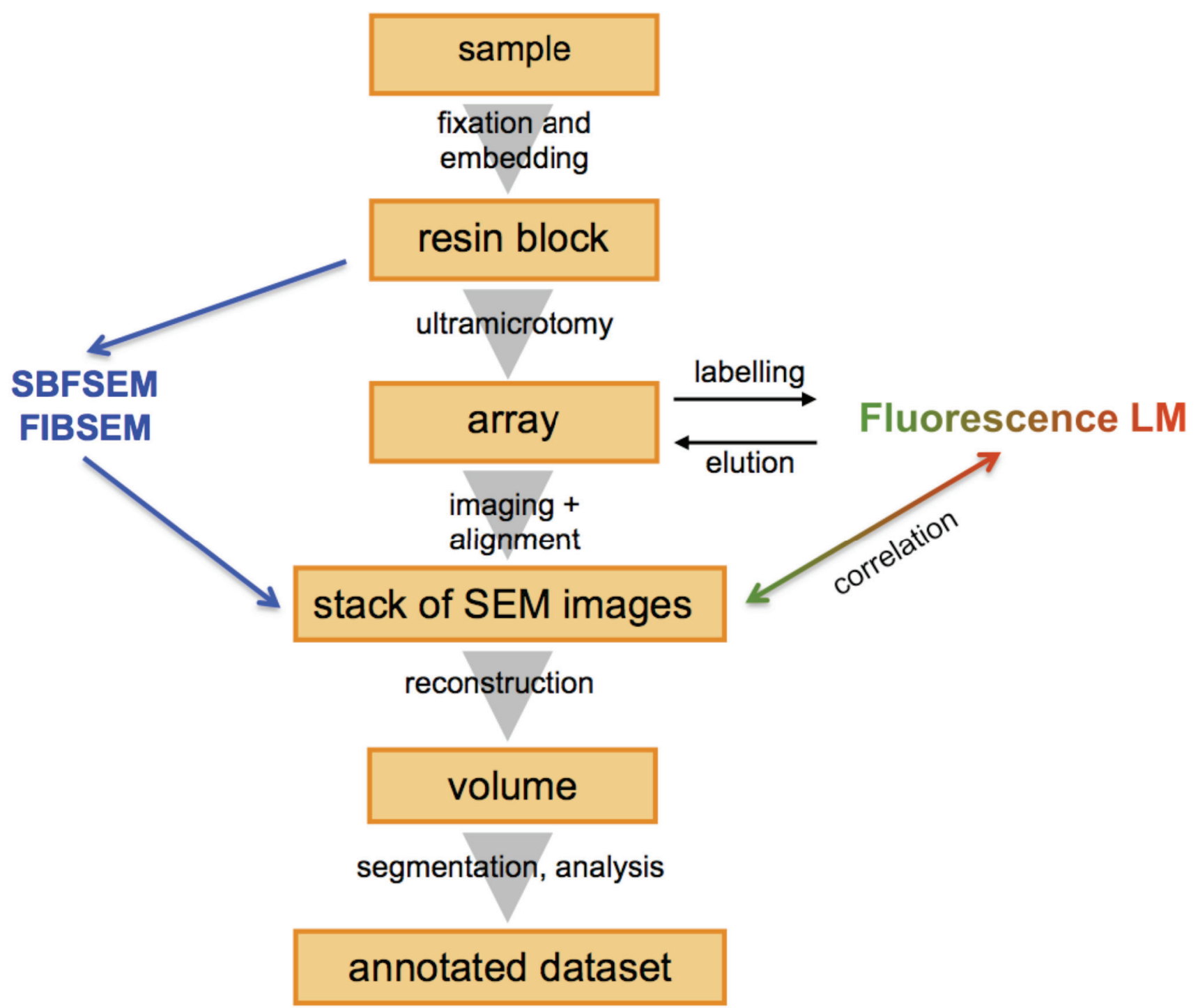

Figure 1: Options for high resolution imaging of tissue in 3D 